\section{A Rapid Cleaning Technique For EM Cathode Assemblies}

Steve Chapman, Protrain, Oxford, England

During a recent one week "Intensive SEM" course that took place in Johannesburg, South Africa, the students came up with a superb cleaning technique that they wished to report upon. The results are, or should be, interesting to everyone who has to clean a cathode assembly! This note was originally posted on MSA's microscopy listserver and generated several replies. I have included more information below in response to these replies.

Often the most time consuming operation during the routine use of a SEM or TEM is the cleaning of a cathode assembly. The procedure, outlined as follows, requires little operator intervention, is free from possible cathode contamination by the cleaning media, and takes comparatively very little time.

\section{History}

There is a vast array of cleaning media used by laboratories to clean the cathode assemblies of electron microscopes. With many of these, the biggest failing is the difficulty in completely removing the cleaning media - which leads to excessive contamination within the system. This problem is further complicated by the human hazards associated with some of the solvents being used. In some countries acetone and ether are not permitted in the laboratory!

I have been using and teaching an ultrasonic cleaning technique developed in 1964. The procedure took advantage of tungsten being soluble in an ammonia solution $\left(\mathrm{NH}_{4} \mathrm{OH}\right)$ and combined this media with any metal polish that was also soluble in ammonia. The technique used an stock ammonia solution diluted down to 10 to 15 parts water to 1 part ammonia. A range of metal polishing media had been used depending on their availability in various countries of the world. This cleaning procedure relied more upon the abrasive effect of the metal polishing media rather than on the chemical attack from the ammonia. Subsequent to the metal polish-ultrasonic cleaning period of about 30 minutes, the polishing medium was removed by way of two further 5 minute ultrasonic cleaning periods in the dilute ammonia alone, to ensure complete removal of the metal polishing medium. The components were then washed in alcohol and dried with a hot air blower. With severely contaminated cathodes, as would be typical of a SEM used at high emission currents, a degree of manual cleaning was often required in the "burnt on" tungsten areas around the cathode aperture. That could be prior to, or after, the initial ultrasonic cleaning procedure.

\section{The New Procedure}

The cathode assembly was placed, aperture facing upwards, in a beaker of stock ammonia solution diluted 3 parts ammonia to one part water and placed in an ultrasonic cleaner. The stock solution was thought to be about $40 \%$ ammonia. After 15 minutes in the ultrasonic cleaner the beaker was placed under running water and thoroughly flushed. Care was taken to ensure that none of the clamping or alignment screws had fallen out of the cathode assembly as they could be flushed away! The cathode was then washed with alcohol before being dried with a hair drier. A new filament was fitted and centered. The assembly was checked for cleanliness by observing with a $20 \mathrm{X}$ lens prior to reinstallation in

\title{
Si(Li)-Detectors
}

stainless steel dewar, UHV technology excellent energy resolution best choice for light element analysis unrivalled long term stability

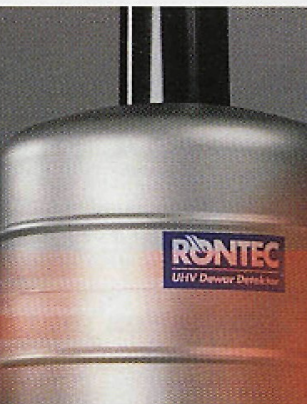

\section{Evaluation Strategy}

standardless analysis on rough surfaces and particles physical calculation of the bremsstrahlung integrated vexpert system《 comprehensive error calculation application-specific automatic quantification

\section{NEW! treme Element Imaging} liquid nitrogen free RÖNTEC XFlash detector 8 times faster than EDX with digital pulse processing combines with any existing EDX $\underset{k 0 p s}{50}$
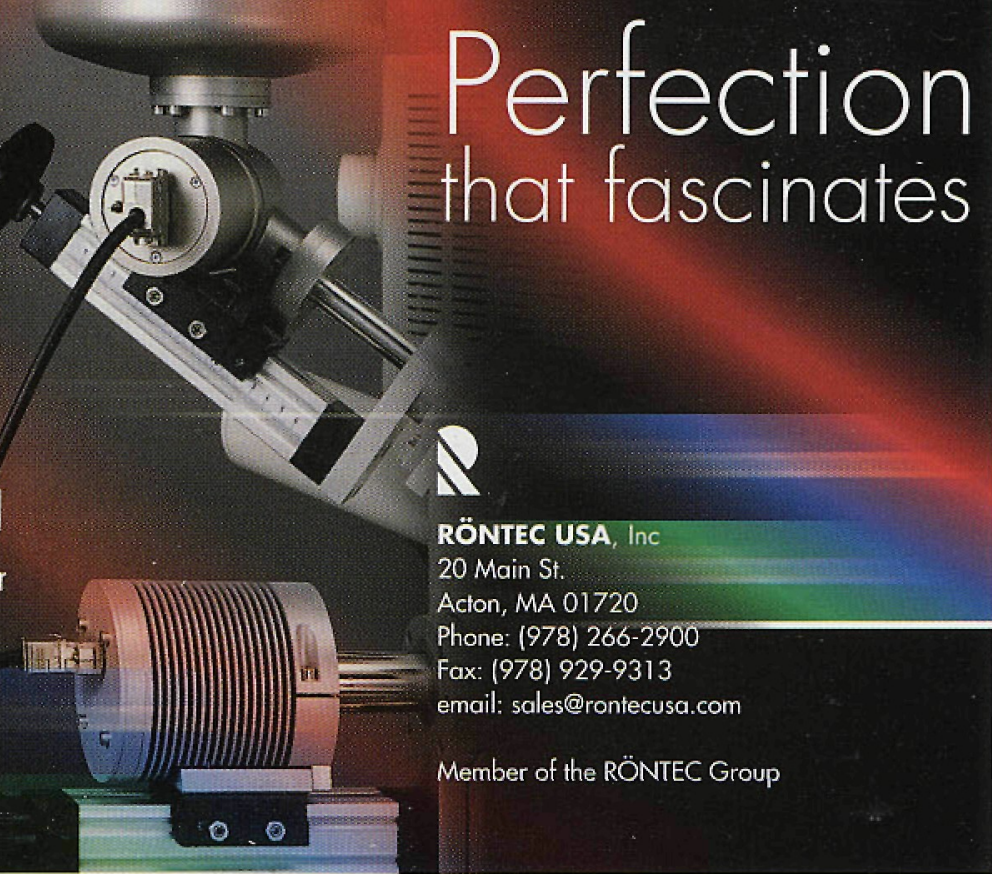

RÖNTEC USA, Inc

20 Main St.

Acion, MA 01720

Phone: (978) 266-2900

Fax: (978) 929-9313

email: sales@rontecusa.com

Member of the RÖNTEC Group 
the microscope. Time for this procedure is less than 25 minutes.

\section{Safety}

Great care was taken not to allow the ammonia solution to make contact with the skin or eyes of the operator. When flushing the solution with water, the flow was set so it would not splash solution on the operator prior to placing the beaker under the flow.

\section{Observations}

The procedure was used on a severely contaminated SEM cathode and a cathode assembly from an electron probe. The contamination rate had been noted as an earlier part of the course, so we are able to state that observations in the SEM before and after cleaning indicated little or no increase in contamination levels.

The above "New Procedure" complements of Errol Kelly (University of Fort Hare), Belinda White (University of Natal, Pietermaritzburg), Allan Hall (University of Pretoria), Akos SzaboRand (Afrikaans University) and Neville Barker (Anaspec).

We were all amazed at the results: a totally wet cleaning method, a perfectly clean cathode assembly with apparently no instrument problems!

Since EM manuals do not mention if parts are aluminum (found in some SEM anodes), tantalum (Philips cathode apertures only), or something else, components should be checked to see how they will react with $\mathrm{NH}_{4} \mathrm{OH}$ before cleaning with this method. This is easily done by placing a very small drop of ammonia solution before going all out with the technique as it is only meant to be used on the stainless steel gun components!

A few points are below in response to those who replied on the microscopy listserver to our request for comment.

1) Three people, two from the USA, one from South Africa already use this technique. They have not seen any problems.
2) There are those who use manual techniques that they claim are far quicker. The point of this technique is to remove the manual component from cleaning, as the methods and media used are often the most common reasons for increased contamination within a column. Hand cleaning methods, particularly electromechanical methods, are also far more damaging to the cathode than a wet technique. Misshaped cathode apertures are found to be the cause of terminal astigmatism! A liquid cleaning is a preferred route as it removes the metal polish and cotton or tissue used to apply the polish. All three media are difficult to remove and may cause contamination and discharge in an electron gun if they are not completely removed. A higher level of technical and observational skills are also required with manual techniques.

3) There are some who have commented that the occasional hand polish, or better still, the ultrasonic cleaner with a metal polish is useful as it removes any irregularities caused by discharge. I agree with them, and perhaps one in three cleans should follow this route in a TEM. There should be less of a problem in a SEM.

4) It has been mentioned that plated cathodes may be damaged if the plating is incomplete. I do not know of any plated cathodes. As far as I have seen, all cathodes are stainless steel, with some using tantalum apertures. Take care if you clean a SEM anode, as some are made of aluminum alloy and they will go black when placed in an ammonia solution!

5) Tantalum, so I am told, is only mildly attacked by alkalis, needing to heated above $150^{\circ} \mathrm{C}$ to encourage unacceptable attack. Thanks to Bill Tivol (Wadsworth Center, NY State Dept. of Health) for that information.

6) The suggestion of using plasma etching as the ideal cleaning device for all components is not challenged, however the ammonia method described is cheap, simple and almost always available in EM laboratory worldwide.

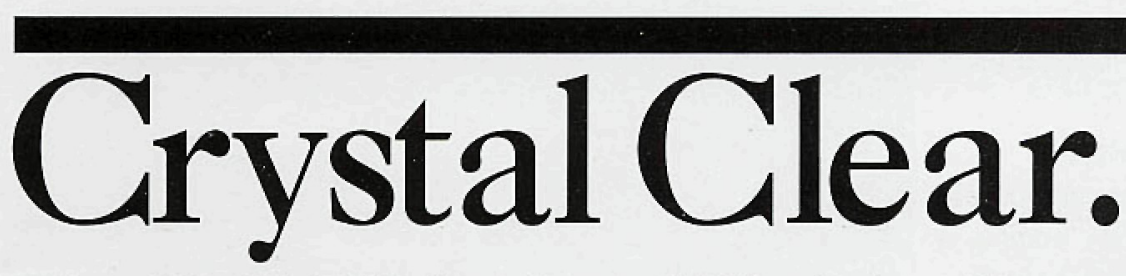

\section{The Meiji EM Series of Modular Stereo Mieroscopes.}

If you are looking for precision, durability, quality and value in a stereo microscope, we invite you to take a closer look at Meiji's EM Series of Stereo Microscopes.

The modular design (A wide variety of bodies, single magnification or zoom- rotatable $360^{\circ}$, auxiliary lenses, eyepieces, stands, holders, etc.) gives you the freedom to create the ideal instrument for your specific needs or application, and Meiji stands behind every instrument with its limited Lifetime Warranty.

For more information on these economically priced stereo microscopes, please call, FAX or write us today.

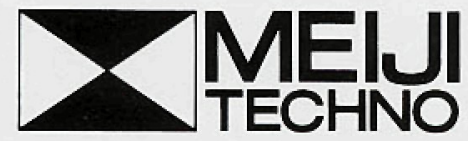

\section{MEIJI TECHNO AMERICA}

2186 Bering Drive, San Jose, CA 95131, Toll Free Telephone: 800.832 .0060 FAX: 408.428.0472, Tel: 408.428.9654 